\title{
Pharyngeal Necrosis, CTCAE
}

National Cancer Institute

\section{Source}

National Cancer Institute. Pharyngeal Necrosis, CT CAE. NCI Thesaurus. Code C57855.

A disorder characterized by a necrotic process occurring in the pharynx. 